\title{
NOTE ON HOMOGENEOUS PLANE CONTINUA
}

\author{
F. BURTON JONES
}

In his Doctoral Dissertation (Texas, 1947), E. E. Moise proved that there exists a compact plane continuum $M$ (not an arc) which is homeomorphic to each of its subcontinua [1]. ${ }^{1}$ Subsequently, R. H. Bing showed that $M$ is homogeneous [2]. Bing's result flatly contradicts the previously announced result of G. Choquet to the effect that a homogeneous, compact, plane continuum must be a simple closed curve [3]. It is the purpose of this note to show that had Choquet assumed in addition to homogeneity that the continuum was aposyndetic ${ }^{2}$ at some point, or that some point of the continuum failed to be a weak cut point ${ }^{3}$ of it, then his conclusion would have been valid.

THEOREM 1. If a compact, plane continuum $M$ is both homogeneous and aposyndetic, then $M$ is a simple closed curve.

Proof. If a point of $M$ is of order 2 in $M$, then $M$ is a simple closed curve [4]. So suppose that no point of $M$ is of order 2 in $M$.

Let $G$ denote the collection of all the complementary domains of $M$. Because $M$ is homogeneous and contains a non-separating point, no point of $M$ separates $M$. Since $M$ is aposyndetic, $M$ is semi-locallyconnected [5]. Hence each element of $G$ is a simple domain $[6,7]$. Let the simple closed curve $J$ denote the boundary of an element $D$ of $G$.

Case 1. If $M-J$ is connected, then each point of $M$ belongs to some such simple closed curve lying in $M$. Consequently each point of $M$ belongs to the boundary of an element of $G$. But $G$ is countable. Hence $M=\sum J_{i}(i=1,2,3, \cdots)$, such that for each $i, J_{i}$ is the boundary of an element of $G$. Since no point of $M$ is of order 2 in $M$, each point of $J_{i}$ is a limit point of $M-J_{i}$. This contradicts a well known theorem (Baire).

Presented to the Society, November 28, 1947; received by the editors March 5, 1948.

${ }^{1}$ Numbers in brackets refer to the bibliography at the end of this paper.

${ }^{2}$ A continuum $M$ is said to be aposyndetic at a point $x$ of $M$ if for each point $y$ of $M-x$, there exists a subcontinuum of $M$ and an open subset $U$ of $M$ such that $M-y \supset H \supset U \supset x$. If a continuum is aposyndetic at each of its points, then it is said to be aposyndetic.

${ }^{3}$ A point $p$ of a continuum $M$ is a weak cut point of $M$ if $M-p$ is not strongly (continuum-wise) connected. For other definitions the reader is referred to Moore's book or Whyburn's book, volumes 13 and 28, respectively, of the American Mathematical Society Colloquium Publications. 
Case 2. If $M-J$ is not connected, then $M-J=H+K$ such that $H \cdot \bar{K}=\bar{H} \cdot K=0$. There exists an arc $T$ lying in the complement of $\bar{D}$ which is irreducible from $H$ to $K$. Except for its end points, $T$ lies in an element $U$ of $G$. Let $C$ denote the boundary of $U$ and let the point $x$ of $C$ be one of the end points of $T$. The component $A x B$ of $C-C \cdot J$ which contains $x$ is an arc-segment whose end points lie on $J$. It follows that $M-(A+B)$ is not connected, but $M-B$ is connected. Hence $A$ is a local separating point of $M$. Since $M$ is homogeneous, every point of $M$ is a local separating point of $M$. But all except countably many of the local separating points of $M$ must be of order 2 in $M$ [8].

Theorem 2. If a homogeneous, compact, plane continuum $M$ contains no weak cut point, it is a simple closed curve.

Proof. If $M$ contains no weak cut point, it is aposyndetic at some point [9]. Being aposyndetic at one point, $M$ must be aposyndetic at each of its points. Hence Theorem 2 follows from Theorem 1.

\section{BIBLIOGRAPHY}

1. E. E. Moise, An indecomposable plane continuum which is homeomorphic to each of its nondegenerate subcontinua. Trans. Amer. Math. Soc. vol. 63 (1948) pp. 581-594.

2. R. H. Bing, A homogeneous indecomposable plane continuum, Bull. Amer. Math. Soc. Abstract 53-5-268.

3. G. Choquet, Prolongement d'homeomorphies. Ensembles topologiquement nommables. Characterisation topologique individuelle des ensembles fermes totalement discontinuum, C. R. Acad. Sci. Paris vol. 219 (1944) pp. 542-544, as reported in Mathematical Reviews vol. 7 (1946) p. 335.

4. Karl Menger, Kurventheorie, Teubner, 1932, p. 267.

5. F. B. Jones, Concerning aposyndetic continua and certain boundary problems, Amer. J. Math. vol. 58 (1941) pp. 545-553, Theorem 4.

6. R. L. Wilder, Sets which satisfy certain avoidability conditions, Casopis pro Pěstováni Mathematiky a Fysiky vol. 67 (1938) pp. 185-198.

7. G. T. Whyburn, Semi-locally-connected sets, Amer. J. Math. vol. 61 (1939) pp. 733-749.

8. G. T. Whyburn, Analytic topology, Amer. Math. Soc. Colloquium Publications, vol. 28, Theorem 9.1, p. 61 .

9. F. B. Jones, Concerning non-aposyndetic continua, Amer. J. Math. vol. 70 (1948) pp. 403-413, Theorem 18.

The University of Texas 